\title{
ERCP in RYGB-patients: One size fits all?
}

\section{(이요 $\circledast \odot$}

\author{
Author \\ Lars Aabakken \\ Institution \\ OUS-RH - Medicine, Oslo, Norway \\ Bibliography \\ DOI https://doi.org/10.1055/a-1161-8755 | \\ Endoscopy International Open 2020; 08: E883-E884 \\ (c) Georg Thieme Verlag KG Stuttgart · New York \\ elSSN 2196-9736
}

\author{
Corresponding author \\ Lars Aabakken, OUS-RH - Medicine, Sognsvannsv. 20 Oslo \\ Oslo 0027, Norway \\ Fax: +23072410 \\ larsaa@medisin.uio.no
}

The obesity epidemic is among the world's most devastating health problems, outnumbering starvation, and even the COVID-19 pandemic in morbidity and mortality. Although weight loss efforts through lifestyle modifications are generally recommended, and do add to the necessary armamentarium, they are by themselves usually insufficient. Conversely, bariatric surgery is well established as an effective treatment, and among the alternative methods, Roux-en-Y gastric bypass (RYGB) is still widely popular, given the current efficacy and acceptable side-effect profile [1].

The altered upper gastrointestinal anatomy in these patients may present various endoscopic challenges. Patients with RYGB anatomy are exposed to hepatobiliary problems, particularly gallstone disease, because of obesity, rapid weight loss, and possibly even because of the blind loop microflora in the excluded duodenal loop with glucuronidase-producing bacteria. Moreover, a substantial proportion of patients present with abdominal pain mandating gastroscopy to exclude gastroduodenal pathology [2]. Thus, endoscopic access to the excluded segment of the gastrointestinal tract is important, but difficult.

The main options for access have been device-assisted enteroscopy (E-ERCP) and laparoscopy-assisted access, primarily for laparoscopy-assisted endoscopic retrograde cholangiopancreatography (LA-ERCP). Both methods are well described and effective, but also fraught with specific challenges. The enteroscopic route to the gastroduodenum is lengthy, and requires time, as well as special skills not widely available. Also, the peripapillary positioning for ERCP is challenging, with acute angulation, limited tip maneuverability, and lack of elevator for catheter manipulation. LA-ERCP, on the other hand, offers easier access to the papilla, and a standard duodenoscope with standard accessories can be used. It does, however, require an experienced laparoscopic surgeon for appropriate trochar placement, along with the time, cost, and logistic challenges of a perioperative ERCP in the operating theatre.
Endoscopic ultrasound-directed transgastric ERCP (EDGE) has emerged as an alternative to the existing suboptimal approaches to pancreatobiliary intervention in these patients. Placement of a temporary lumen-apposing metal stent (LAMS) into the excluded stomach was initially described in 2014 [3], as a means of accessing the papilla through a close-to-normal route with a duodenoscope for pancreatobiliary endoscopy. The method obviates the lengthy route and positional challenges of E-ERCP, as well as the logistics and specific complications of LA-ERCP, allowing an endoscopist facile in EUS and ERCP to complete the procedure within the endoscopy unit.

Naturally, the three methods are alternatives that need comparative analysis. A recent meta-analysis addressed the issue [4], and concluded that the technical and clinical success of EDGE is better than that of E-ERCP and comparable to that of LA-ERCP, with adverse events (AEs) similar to that of the laparoscopic approach. At the moment, however, available data are all based on retrospective case series, where direct comparisons remain difficult.

The series by Kochar et al in this edition of EIO [5] is a welcome addition to the existing literature. They present a 56-patient, single-center, 4-year retrospective experience with all three methods, with 26, 18, and 12 patients handled by EDGE, LA-ERCP, and E-ERCP, respectively. Their results mirror that of the general literature quite well, with superior technical success with EDGE and LA-ERCP compared to E-ERCP. Methodspecific AEs were minimal in this study, but numbers were too low for adequate comparison. The relative safety of E-ERCP suggested by a recent meta-analysis probably still holds true [4].

More importantly, however, the authors suggest an algorithm for assigning patients to each method, which to me makes a lot of sense at the current time. It does mandate the availability of all three options, but that would likely often be the case in a referral center. Their algorithm also underlines that the methods are complementary, rather than competing 
alternatives. Briefly, a concomitant indication for cholecystectomy would favor LA-ERCP, while patients with surgical anatomy amenable to E-ERCP should be referred for that. EDGE remains reserved for patients without need for cholecystectomy and with impossible or failed enteroscopic access.

This seems a very reasonable conclusion based on current knowledge, and counters some single-method enthusiast reports available. It is not clear whether this algorithm was, in fact, applied by the authors; likely it has emerged over time with increasing experience with all methods.

While EDGE may indeed turn out to be the preferred method in this situation, questions remain that are only partially addressed by the current paper:

For now, the method is still non-standardized, and even the report by Kochhar describes various variants. Questions about aspects such as stent caliber, placement techniques (need for guidewire, stent fixation, multi-step procedures), significance of complications such as persistent fistula or stent dislodgment, and cost considerations remain open and mandate careful consideration. With increasing general expertise in placing LAMS for other indications, likely the rates of AE will decrease for this procedure as well, but the specific issues of dislodgement due to endoscope traversing must be addressed. The safest strategy appears to be a two-step procedure to allow maturation of the tract before ERCP, if it is clinically feasible. In most situations, a separate procedure would be needed for delayed stent removal anyway. However, urgent cases may mandate a different strategy, and the current method may not be optimal for a one-step procedure. Perhaps t-tag fixation of the anastomosis would be worth exploring, or improved fixation of the LAMS? These questions need to be addressed before the EDGE procedure is introduced into general practice. The current rate of $A E s$ is a concern, and expansion of the technique is likely to increase this rate further. Refinement of the technique in the hands of experts is likely a prudent way forward.

On the other hand, EDGE has additional benefits that must be acknowledged. In particular, with EDGE, EUS-guided proce- dures can be performed that would be difficult or impossible with enteroscopy- or even laparoscopy-assisted procedures [6]. This utility further adds to the reasons for developing this EUS-based shortcut for anatomical access.

This paper underlines the expanding role of combined EUS/ ERCP utility needed in advanced pancreatobiliary endoscopy units, and the authors are to be commended for moving the field one more step forward in this respect.

\section{Competing interests}

The authors declare that they have no conflict of interest.

References

[1] Morales-Maza J, Rodriguez-Quintero JH, Sanchez-Morales GE et al. Laparoscopic Roux-en-Y gastric bypass in the treatment of obesity: evidence based update through randomized clinical trials and metaanalyses. Il Giornale di chirurgia 2020; 41: 5-17

[2] Blom-Hogestol IK, Stubhaug A, Kristinsson JA et al. Diagnosis and treatment of chronic abdominal pain 5 years after Roux-en-Y gastric bypass. Surg Obes Relat Dis 2018; 14: 1544-1551

[3] Kedia P, Sharaiha RZ, Kumta NA et al. Internal EUS-directed transgastric ERCP (EDGE): game over. Gastroenterology 2014; 147: 566-568

[4] Dhindsa BS, Dhaliwal A, Mohan BP et al. EDGE in Roux-en-Y gastric bypass: How does it compare to laparoscopy-assisted and balloon enteroscopy ERCP: a systematic review and meta-analysis. Endosc Int Open 2020; 8: E163-E171

[5] Kochhar G, Mohy-ud-din N, Grover A et al. EUS Directed transgastric endoscopic retrograde cholangio-pancreatography versus laparoscopic-assisted ERCP versus deep enteroscopy assisted ERCP. Endosc Int Open 2020; 08: E877-E882

[6] Krafft MR, Hsueh W, James TW et al. The EDGI new take on EDGE: EUS-directed transgastric intervention (EDGI), other than ERCP, for Roux-en-Y gastric bypass anatomy: a multicenter study. Endosc Int Open 2019; 7: E1231-E1240 